\title{
Image Denoising Using Euler-Lagrange Equations for Function-Valued Mappings
}

\author{
Daniel Otero ${ }^{1}$, Davide La Torre ${ }^{1,2,3}$, and Edward R. $\operatorname{Vrscay}^{1(\bowtie)}$ \\ 1 Department of Applied Mathematics, Faculty of Mathematics, \\ University of Waterloo, Waterloo, ON N2L 3G1, Canada \\ \{dotero, ervrscay\}@uwaterloo.ca \\ 2 Department of Economics, Management, \\ and Quantitative Methods, University of Milan, Milan, Italy \\ davide.latorre@unimi.it \\ 3 Department of Applied Mathematics and Sciences, \\ Khalifa University, Abu Dhabi, UAE \\ davide.latorre@kustar.ac.ae
}

\begin{abstract}
In this paper, we consider a new method for representing complex images, e.g., hyperspectral images and video sequences, in terms of function-valued mappings (FVMs), also known as Banach-valued functions. At each (pixel) location $x$, the FVM image $u(x)$ is a function, as opposed to the traditional vector approach. We define the Fourier transform of an FVM as well as Euler-Lagrange conditions for functionals involving FVMs and then show how these results can be used to devise some FVM-based methods of denoising. We consider a very simple functional and present some numerical results.
\end{abstract}

\section{Introduction}

Vector-valued functions provide a quite natural representation for some types of images, for example, colour and hyperspectral (HS) images [4,11]. Furthermore, many efficient vector-based imaging tools have been introduced [2-4,7]. That being said, the vector-valued approach has some limitations when it comes to model the internal structure of certain complex data sets. For instance, let us consider a video sequence, which can be represented as a vector-valued function

$$
u: \Omega \rightarrow \mathbb{R}^{N} .
$$

Here, $\Omega \subset \mathbb{R}^{2}$ or $\mathbb{R}^{3}$ is the spatial domain, and each component $u_{i}$ of the range of $u, 1 \leq i \leq N$, is a time frame. Proposing a suitable space of functions as a suitable model for the temporal functions defined at each pixel of the video is not possible due to the finite dimensionality of the range of $u$. A similar situation exists in the case of HS images - here it is important to recall that the entries $u_{i}(x)$ comprising an HS image represent a discretization or sampling of the continuously-defined spectral function at $x$.

In this paper, to overcome the difficulty of modelling the internal structure of complex data sets using vector-valued functions, we propose to "unvectorize"

(C) Springer International Publishing Switzerland 2016

A. Campilho and F. Karray (Eds.): ICIAR 2016, LNCS 9730, pp. 110-119, 2016.

DOI: $10.1007 / 978-3-319-41501-7 \_13$ 
them, i.e., consider the variable $y$ in temporal or spectral space to be continuous as opposed to discrete. As a result, an image $u$ is represented by a function-valued mapping (FVM). Briefly, an FVM is a mapping of the form:

$$
u: X \rightarrow \mathcal{F}(Y)
$$

where $X$ is the support of the FVM (for digital images, the "pixel space") and $\mathcal{F}(Y)$ is a Banach space of either real- or complex-valued images supported on the set $Y$. In other words, at each $x \in X$, the "value" of the image function $u(x)$ is a function which belongs to a space $\mathcal{F}(Y)$ that is appropriate to the application. For example, an HS image could be represented as a FVM of the form,

$$
u: X \subset \mathbb{R}^{2} \rightarrow L^{2}(\mathbb{R})
$$

where $L^{2}(\mathbb{R})$ is the space of square-integrable functions supported on the real line, i.e., spectral functions with finite energy.

Of course, it remains to develop appropriate methods and tools which can operate on FVMs. In this paper, we define Fourier transforms and an EulerLagrange equation for FVMs which are then employed to denoise HS images.

It is important to mention that the FVM approach is not a novelty in other fields such as partial differential equations [20], harmonic analysis [15,17], statistics [1], and others [8]. Indeed, FVMs are known in the mathematical community as Banach-valued functions, the latter being studied mainly by analysts who have been interested in seeing if the classical results of real-valued functions still hold in the Banach-valued setting $[6,8]$.

In imaging science, however, this methodology has been barely explored. Nevertheless, some contributions can be found which employ the concept of a function which assumes values in an infinite-dimensional Banach space. For example, in an effort to close the gap between the mathematical formalism of Banach-valued functions and practical applications in imaging, the authors in [14] use the FVM approach to provide a solid mathematical platform to describe and treat diffusion magnetic resonance images. Also, in [13], an analogue of FVMs is introduced, namely, measure-valued images, which are well suited for non-local image processing. As a matter of fact, non-local means denoising [5] and fractal image coding [12] are the two applications that are addressed in [13] using this measure-valued methodology.

Rather than introducing state-of-the-art algorithms for image denoising, the main purpose of this paper is to present the FVM approach as a mathematical framework that may offer interesting possibilities for the image processing community. That being said, we do present a couple of computational examples that illustrate both the novelty and the potential of FVMs.

\section{Function-Valued Mappings}

An FVM is a particular case of a mapping defined between two Banach spaces. Many definitions and properties that are valid in the real case can be extended 
without difficulties to this setting (see $[1,6,8,16,20]$ for more details). In this section, we present a definition of the Fourier transform for FVMs, as well as an Euler-Lagrange equation for a certain type of functionals whose argument involve these kind of mappings.

\subsection{Fourier Transform}

In [17], Peetre provides perhaps one of the first generalizations of the Fourier transform for Banach-valued functions. In fact, for $p \in(1,2]$, Peetre proves that the Fourier transform is a bounded operator from $L^{p}(\mathbb{R} ; Z)$ to $L^{q}(\mathbb{R} ; Z)$, where $q$ is the Hölder conjugate of $p$ and $Z$ is a Banach space. This result was extended further by Milman in [15]. Along these lines, in [18], as an application of the Bochner integral [1], the following definition of the Fourier transform is provided for Banach-valued functions that belong to $L^{1}\left(\mathbb{R}^{n} ; H\right)$, where $H$ is a separable Hilbert space:

$$
U(\omega):=\int_{\mathbb{R}^{n}} e^{i \omega \cdot x} u(x) d x,
$$

Note that in [18], the exponent of the complex exponential has a positive sign. Moreover, it is shown that $U(\omega)$ is well defined and that it is a bounded operator from $L^{1}\left(\mathbb{R}^{n} ; H\right)$ to $L^{\infty}\left(\mathbb{R}^{n} ; H\right)$. However, no definition of the inverse Fourier transform is presented.

The existing definitions of the Fourier transforms for Banach-valued functions provide the foundation for defining the Fourier transform of FVMs. In particular, we focus our attention on the elements of the space $L^{1}\left(\mathbb{R}^{n} ; \mathcal{F}(Y)\right)$; that is, the space of integrable FVMs.

Definition 1. Let $u \in L^{1}\left(\mathbb{R}^{n} ; \mathcal{F}(Y)\right)$, where $\mathcal{F}(Y)$ is a complex-valued space. We define the Fourier transform of $u$ as the integral

$$
\mathbf{F}(u)(\omega):=\int_{\mathbb{R}^{n}} e^{-i \omega \cdot x} u(x) d x,
$$

where $\omega \in \mathbb{R}^{n}$. In some cases, we will denote $\mathbf{F}(u)(\omega)$ as $U(\omega)$ as well.

Theorem 1 [18]. F is a bounded operator of the form

$$
\mathbf{F}: L^{1}\left(\mathbb{R}^{n} ; \mathcal{F}(Y)\right) \rightarrow L^{\infty}\left(\mathbb{R}^{n} ; \mathcal{F}(Y)\right) .
$$

Regarding the inverse transform, this operator is not well defined for all the elements that belong to $L^{\infty}\left(\mathbb{R}^{n} ; \mathcal{F}(Y)\right)$ since not all the FVMs of this space are in $L^{1}\left(\mathbb{R}^{n} ; \mathcal{F}(Y)\right)$. Given this, as is customary in harmonic analysis [9], we define this transform under the assumption that both $u$ and $U$ belong to $L^{1}\left(\mathbb{R}^{n} ; \mathcal{F}(Y)\right)$.

Definition 2. If both $u$ and $U$ are elements of $L^{1}\left(\mathbb{R}^{n} ; \mathcal{F}(Y)\right)$, we define the inverse Fourier transform of $U$ as

$$
\mathbf{F}^{-1}(U)(x):=\frac{1}{(2 \pi)^{n}} \int_{\mathbb{R}^{n}} e^{i \omega \cdot x} U(\omega) d \omega .
$$


Theorem 2 [16]. If both $u$ and $U$ belong to $L^{1}\left(\mathbb{R}^{n} ; \mathcal{F}(Y)\right)$, then

$$
u(x)=\frac{1}{(2 \pi)^{n}} \int_{\mathbb{R}^{n}} e^{i \omega \cdot x} U(\omega) d \omega .
$$

It is clear that the operator $\mathbf{F}$ is linear, which is, of course, a consequence of the linearity of the integral of the Fourier transform. Let $\alpha, \beta \in \mathbb{C}$ and $u, v \in$ $L^{1}\left(\mathbb{R}^{n} ; \mathcal{F}(Y)\right)$. We claim, without proof, that the following equality holds:

$$
\mathbf{F}(\alpha u+\beta v)=\alpha \mathbf{F}(u)+\beta \mathbf{F}(v) .
$$

As expected, linearity also holds for the inverse operator $\mathbf{F}^{-1}$. Let $\alpha, \beta \in \mathbb{C}$ and $U, V \in L^{1}\left(\mathbb{R}^{n} ; \mathcal{F}(Y)\right)$. Then,

$$
\mathbf{F}^{-1}(\alpha U+\beta V)=\alpha \mathbf{F}^{-1}(U)+\beta \mathbf{F}^{-1}(V) .
$$

As with the classical Fourier transform, in the FVM setting, we also have properties such as translation, scaling, modulation, differentiation with respect to $x$ and integration. These are presented in the following theorems.

Theorem 3 [16]. Let both $u \in L^{1}\left(\mathbb{R}^{n} ; \mathcal{F}(Y)\right)$. Also, let $\omega_{0}, x_{0} \in \mathbb{R}^{n}$ and $a \in \mathbb{R}$, $a \neq 0$. Then, the following assertions hold:

1. Translation: $\mathbf{F}\left(u\left(x-x_{0}\right)\right)(\omega)=e^{-i \omega \cdot x_{0}} U(\omega)$.

2. Modulation: $\mathbf{F}\left(e^{-i \omega_{0} \cdot x} u(x)\right)(\omega)=U\left(\omega-\omega_{0}\right)$.

3. Scaling: $\mathbf{F}(u(a x))(\omega)=\frac{1}{|a|^{n}} U\left(\frac{\omega}{a}\right)$.

4. Integration: $\int_{\mathbb{R}^{n}} u(x) d x=U(0)$.

Theorem 4 [16]. Assume all $\frac{\partial^{l} u}{\partial x_{j}^{l}}$ and $\left(i \omega_{j}\right)^{l} U$ are elements of $L^{1}\left(\mathbb{R}^{n} ; \mathcal{F}(Y)\right)$ whenever $0 \leq l \leq k$. Then,

$$
\mathbf{F}\left(\frac{\partial^{k} u}{\partial x_{j}^{k}}\right)(\omega)=\left(i \omega_{j}\right)^{k} \mathbf{F}(u)(\omega) .
$$

\subsection{The Euler-Lagrange Equation}

We simply present the Euler-Lagrange equation of a given functional whose argument is a FVM of the form $u: X \subset \mathbb{R}^{n} \rightarrow \mathcal{F}(Y)$. In particular, we focus our attention on the following type of functionals:

$$
I(u)=\int_{X} f\left(x, u(x), \nabla_{x} u(x)\right) d x,
$$

where $f: X \times \mathcal{F}(Y) \times \mathcal{G}^{n}(Y) \rightarrow \mathbb{R}$ is a mapping that is Fréchet differentiable with respect to all of its arguments, and $\mathcal{G}^{n}(Y)$ is the Cartesian product of the range of $\nabla_{x} u$; that is, $\mathcal{G}^{n}(Y)=\mathcal{G}(Y) \times \cdots \times \mathcal{G}(Y)$, where $\frac{\partial u}{\partial x_{i}}: X \subset \mathbb{R}^{n} \rightarrow \mathcal{G}(Y)$. As expected, the solution of the Euler-Lagrange equation is a FVM that belongs to the set of stationary points of $I(u)$. 
Theorem 5 [16]. Let $(X, \Sigma, \mu)$ and $(Y, T, \nu)$ be finite measure spaces. Also, let $u: X \subseteq \mathbb{R}^{n} \rightarrow \mathcal{F}(Y), \frac{\partial u}{\partial x_{i}}: X \subset \mathbb{R}^{n} \rightarrow \mathcal{G}(Y)$, and assume that the function

$$
\Phi(x):=f\left(x, u(x), \nabla_{x} u(x)\right)
$$

is integrable over $X$. In addition, suppose that the Fréchet derivatives of $f$ : $X \times \mathcal{F}(Y) \times \mathcal{G}^{n}(Y) \rightarrow \mathbb{R}$ with respect to all of its arguments are continuous. Define the functional $I(u): Z(\mathcal{F}(Y), \mathcal{G}(Y)) \rightarrow \mathbb{R}$ as follows:

$$
I(u):=\int_{X} f\left(x, u(x), \nabla_{x} u(x)\right) d x
$$

where $Z(\mathcal{F}(Y), \mathcal{G}(Y))$ is a Banach space of $F V M$ s that depends on the function spaces $\mathcal{F}(Y)$ and $\mathcal{G}(Y)$. If $u_{0}: X \subset \mathbb{R}^{n} \rightarrow \mathcal{F}(Y)$ is a stationary point of $I(u)$, $u_{0}$ is the solution of the equation

$$
\frac{\partial f}{\partial u}\left(u_{0}\right)-\nabla \cdot \frac{\partial f}{\partial \nabla_{x} u}\left(\nabla_{x} u_{0}\right)=0 .
$$

where $\frac{\partial f}{\partial u} \in \mathcal{F}(Y)^{*}$ and $\frac{\partial f}{\partial \nabla_{x} u} \in \mathcal{G}^{n}(Y)^{*}$ are the Fréchet derivatives of $f$ with respect to $u$ and $\nabla_{x} u$ respectively, $\nabla \cdot$ is the classical divergence operator, and $\mathcal{F}(Y)^{*}$ and $\mathcal{G}(Y)^{*}$ are the dual spaces of $\mathcal{F}(Y)$ and $\mathcal{G}(Y)$ respectively.

We consider Eq. (15) as the Euler-Lagrange equation of the functional $I(u)$ defined in (14). As its classical counterpart, it is also a necessary condition for the solutions of the variational problem stated in the previous theorem. However, it is not a sufficient condition for the existence of such solutions. To determine if such solutions exist, the standard sufficient conditions from calculus of variations can be employed to such an end [20].

\section{A Simple FVM-Based Denoising Method}

In this section we describe one possible denoising method which employs a very simple functional to be minimized. Our motivation was to be able to use both the Euler-Lagrange equations as well as Fourier transforms to solve the minimization problem. We begin with the assumption that the HS images belong to $C^{2}\left(X ; L^{2}(Y)\right)$, where $X \subset \mathbb{R}^{2}$ and $Y \subset \mathbb{R}$. Of course, this is a quite strong - and "unrealistic" - requirement on the HS image in the spatial direction: piecewise $C^{2}\left(X ; L^{2}(Y)\right)$ would be more "realistic." Our assumption allows us to employ the formulation of the Euler-Lagrange equation presented earlier, where all derivatives are understood to be defined in the classical sense. Our regularity assumption, however, can be weakened by using the weak formulation of the EulerLagrange equation, which is beyond the scope of this conference paper.

Under this regularity assumption, we propose to recover a denoised reconstruction $\bar{u}$ of an HS image from a noisy observation $f$ by minimizing the following functional $I: C^{2}\left(X ; L^{2}(Y)\right) \rightarrow \mathbb{R}:$

$$
\min _{u}\left\{\frac{1}{2} \int_{X}\|\rho(u(x)-f(x))\|_{2}^{2} d x+\int_{X}\left\|\nabla_{x} u(x)\right\|_{2}^{2} d x\right\} .
$$


The squared $L^{2}$ norm of the gradient - a kind of "elastic bending" term - is not an optimal norm in terms of denoising ability: An $L^{1}$ norm of the gradient (total variation) would be more effective. The squared $L^{2}$ norm was chosen because it yields a closed-form solution in terms of Fourier transforms.

Also note that we allow the regularization parameter $\rho=\rho(y)$ to be a function of the spectral parameter $y \in Y$, allowing denoising to be performed with different intensities across the spectral domain.

From Sect. 2.2, the Euler-Lagrange equation which corresponds to the functional in Eq. (16) is given by

$$
\int_{Y}\left(\rho(u(x)-f(x))-\frac{\partial^{2} u(x)}{\partial x_{1}^{2}}-\frac{\partial^{2} u(x)}{\partial x_{2}^{2}}\right)(y) d y=0,
$$

Let us recall that the Euler-Lagrange equation states a necessary optimality condition for optimality. Among all possible solutions to Eq. (16) we consider a particular solution $\bar{u}$ such that

$$
\left[\frac{\partial^{2} \bar{u}(x)}{\partial x_{1}^{2}}+\frac{\partial^{2} \bar{u}(x)}{\partial x_{2}^{2}}\right](y)-[\rho(\bar{u}(x)-f(x)](y)=0
$$

for a.e. $x \in X$ and $y \in Y$. Such a $\bar{u}$ may be found by means of the Fourier transform. If we let $F(\omega)=\mathbf{F}\{f\}(\omega)$ denote the Fourier transform of $f$, then

$$
\bar{u}(y)=\mathbf{F}^{-1}\left\{\left[\frac{\rho(y)}{\|\omega\|_{2}^{2}+\rho(y)}\right] F(\omega)\right\} .
$$

In the special case that $\rho(y)=\rho_{0}$, a constant, Eq. (19) becomes

$$
\bar{u}(y)=\mathbf{F}^{-1}\left\{\left[\frac{\rho_{0}}{\|\omega\|_{2}^{2}+\rho_{0}}\right] F(\omega)\right\},
$$

which is the classical low-pass filter result. Because of the simplicity of the model in (16), i.e., no coupling between spectral components with different $y$-values, the filtering in both (19) and (20) may be performed on each $y$-value independently. Reversing the roles of the domains $X$ and $Y$ : We may also consider HS images as mappings from the spectral domain $X \subset \mathbb{R}$ to the space $L^{2}(Y)$, where $Y \subset \mathbb{R}^{2}$. That is, associated with each spectral value $x \in X$ there is a $2 \mathrm{D}$ spatial image function $u(x)$. In this case, the relevant denoising functional is

$$
\min _{u}\left\{\frac{1}{2} \int_{X}\|\rho(u(x)-f(x))\|_{2}^{2} d x+\int_{X}\left\|u^{\prime}(x)\right\|_{2}^{2} d x\right\},
$$

where $u^{\prime}$ is the (classical) derivative of the FVM $u$ (see [16] for details). The procedure described earlier also holds for this representation. In particular, if $\rho: Y \subset \mathbb{R}^{2} \rightarrow \mathbb{R}$, a solution for the corresponding Euler-Lagrange equation has the same form as in Eq. (19), namely,

$$
\bar{u}(y)=\mathbf{F}^{-1}\left\{\left[\frac{\rho(y)}{\omega^{2}+\rho(y)}\right] F(\omega)\right\} .
$$




\section{Numerical Examples}

In our experiments, we assume, for the sake of simplicity, that the power of the noise is constant over the entire HS data set. Also, we assume that the noise is Additive White Gaussian Noise (AWGN). In the discrete context, we consider HS images as 3-D data sets of size $M \times N \times P$; that is, a HS image is a collection of $P$ bands or channels each one of size $M \times N$. Here we have employed two HS images, namely, Indian Pines and Salinas-A, the latter being a subset of the Salinas HS image - both of them can be downloaded from [10]. The sizes of the 3 -D Indian Pines and Salinas- $A$ data sets are $145 \times 145 \times 220$ and $83 \times 86 \times 224$, respectively. AWGN was added to these HS data (assumed to be noiseless). In all experiments, the Peak Signal-to-Noise Ratio (PSNR) before denoising was $30.103 \mathrm{~dB}$.

As expected, we denoise HS images by means of either Eq. (19) or Eq. (22). Clearly, these equations yield continuous FVMs $u$, nevertheless, discrete approximations of such $u$ can be obtained by means of the fast Fourier transform. This can be done easily by noticing that, in a discrete setting, Eq. (19) is equivalent to filtering each band of a noisy HS image independently, whereas Eq. (20) is analogous to denoising each spectral function in an independent fashion. In what follows, we refer to the filterings yielded by both Eq. (19) and Eq. (20) as the "SPATIAL" and "SPECTRAL" methods respectively. As expected, the strength of the denoising process for either each band or spectrum is determined by the function $\rho(y)$, which we obtained experimentally so that the performance of this approach is optimal in the Mean Squared Error (MSE) sense. Also, we consider the case in which $\rho$ is constant across either the spatial or the spectral domains. Once again, we determine $\rho$ experimentally for optimal performance w.r.t. the MSE.

Regarding measures of performance, we employed the Peak Signal-to-Noise Ratio (PSNR) and the Structural Similarity Index Measure (SSIM) [19]. For the latter, we computed the SSIM between the original and recovered HS images in both the spatial and spectral domains. In the spatial case, the SSIM is computed between bands, but in the spectral case the SSIM is computed between spectra. In each of these cases, an overall SSIM is obtained in the usual way-by simply averaging over all computed local SSIMs. A summary of the numerical results is shown in Table 1.

From these results, we observe that denoising with different intensities over either the spatial or spectral domains improves the performance of the proposed FVM approach. This should not be surprising since, in general, the PSNR is not constant across bands and spectra. Having a "regularizing function" $\rho(y)$ may therefore be deemed a good strategy for denoising purposes. Also, it is worthwhile to mention that changing the way in which HS images are represented has also an impact on the obtained reconstructions. Considering HS as FVMs that assign an image to each wavelength value seems to benefit the SPECTRAL denoising methodology. This may be due to the greater regularity that HS images tend to have throughout the spectral domain which, in turn, appears to validate, at least up to a certain extent, the choice of the space $C^{2}\left(X ; L^{2}(Y)\right)$ 
Table 1. Numerical results w.r.t. different image distances. The PSNR prior to denoising was $30.103 \mathrm{~dB}$. The results presented in the second and fourth columns correspond to the cases in which the regularizing parameter is constant. The third and fifth columns show how the performance of the proposed methods is improved when the strength of the denoising process is allowed to vary across either the spatial or spectral domains.

\begin{tabular}{|c|c|c|c|c|}
\hline & \multicolumn{2}{|c|}{ SPATIAL } & \multicolumn{2}{|c|}{ SPECTRAL } \\
\hline & $\rho_{0}$ & $\rho(y)$ & $\rho_{0}$ & $\rho(y)$ \\
\hline \multicolumn{5}{|l|}{ SALINAS-A } \\
\hline PSNR (dB) & 33.1069 & 36.3229 & 34.1434 & 34.3676 \\
\hline SPATIAL SSIM & 0.9008 & 0.9311 & 0.8125 & 0.8254 \\
\hline SPECTRAL SSIM & 0.9872 & 0.9927 & 0.9874 & 0.9877 \\
\hline \multicolumn{5}{|l|}{ INDIAN PINES } \\
\hline PSNR (dB) & 31.5657 & 34.4031 & 33.1165 & 33.1600 \\
\hline SPATIAL SSIM & 0.7368 & 0.8906 & 0.8035 & 0.8054 \\
\hline SPECTRAL SSIM & 0.9873 & 0.9934 & 0.9906 & 0.9907 \\
\hline
\end{tabular}

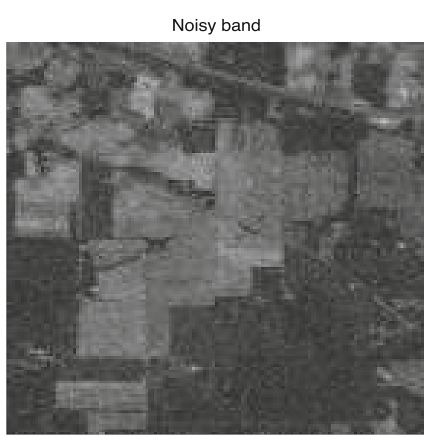

Original band

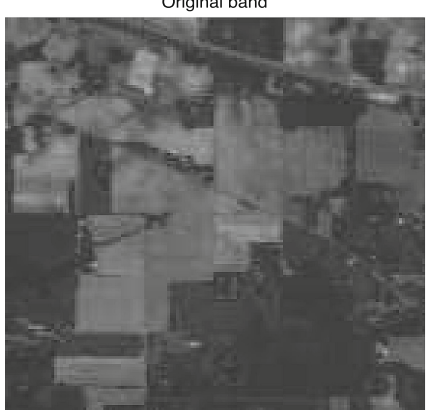

SSIM map

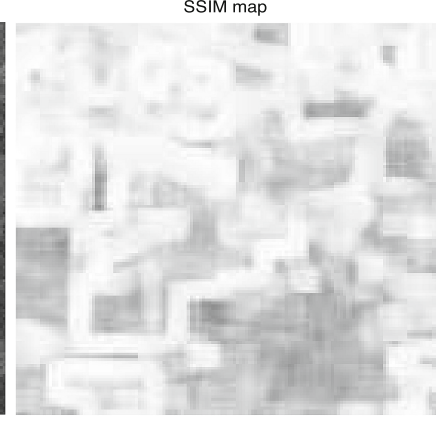

Recovered band

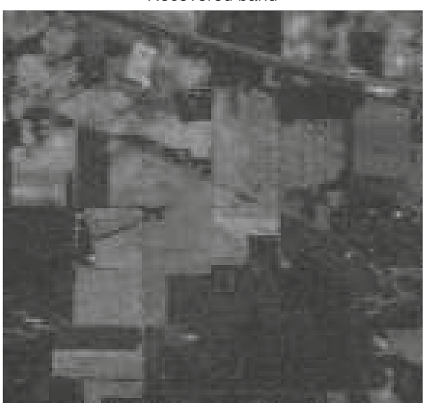

Fig. 1. Visual results for Band No. 23 of the Indian Pines image. Top left: Noisy band. Bottom left: Original (noiseless) band. Bottom right: Reconstructed (denoised) band. Top right: SSIM map between reconstructed (denoised) and original band. 

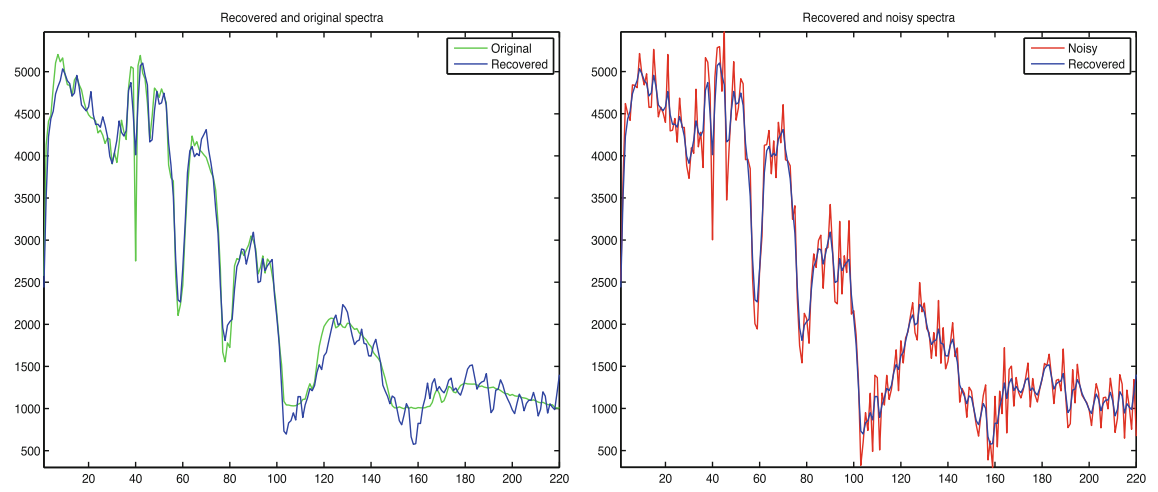

Fig. 2. Results of SPECTRAL denoising method for a particular spectral function of the Indian Pines image. Left: Original (noiseless) spectrum (green) and reconstructed spectrum (blue). Right: Noisy spectrum (red) and reconstructed spectrum (blue). (Color figure online)

as a reasonable model for HS images. Lastly, it can be noticed that the SPATIAL method employing the regularization function $\rho !: Y \subset \mathbb{R} \rightarrow \mathbb{R}$ yields the best performance. This may be due to the fact that several bands of the noisy HS images that were considered in these experiments have a high signal-to-noise ratio, which is an advantage for this method.

Visual results are presented in Figs. 1 and 2. Figure 1 shows how the SPECTRAL method carries out the denoising in the spatial domain - in this case, $\rho: \mathbb{R}^{2} \rightarrow \mathbb{R}$. The SSIM map, shown at the top right of Fig. 1, illustrates the similarity between the reconstruction (denoised) and the original (noiseless) HS data for a particular band. The brightness in these maps is proportional to the magnitude of the local SSIM, i.e., the brighter a given location, the greater the similarity between the denoised and the original bands at that location [19].

Figure 2 shows an example of the denoising process performed by the aforementioned method in the spectral domain. A comparison between a particular reconstruction (blue) and the corresponding noisy spectrum (red) is shown on the left. On the right, the original spectrum (green) along with the corresponding reconstruction (blue) are shown for comparison.

Acknowledgements. This research has been supported in part by the Natural Sciences and Engineering Research Council of Canada (NSERC) in the form of a Discovery Grant (ERV). Financial support from the Faculty of Mathematics and the Department of Applied Mathematics (DO) is also gratefully acknowledged. 


\section{References}

1. Aliprantis, C.D., Border, K.C.: Infinite Dimensional Analysis: A Hitchhiker's Guide. Springer, Heidelberg (2006)

2. Bach, F., Jenatton, R., Mairal, J., Obozinski, G.: Convex optimization with sparsity-inducing norms. In: Sra, S., Nowozin, S., Wright, S.J. (eds.) Optimization for Machine Learning, pp. 19-53. MIT Press, Massachusetts (2012)

3. Beck, A., Teboulle, M.: A fast iterative shrinkage-thresholding algorithm for linear inverse problems. SIAM J. Imaging Sci. Arch. 2, 183-202 (2009)

4. Bresson, X., Chan, T.F.: Fast dual minimization of the vectorial total variation norm and applications to color image processing. Inverse Probl. Imaging 2, 455-484 (2008)

5. Buades, A., Coll, B., Morel, J.-M.: A non-local algorithm for image denoising. In: Proceedings of the Conference on Computer Vision and Pattern Recognition, pp. 60-65. IEEE (2005)

6. Cartan, H., Cartan, H.P.: Differential Calculus. Hermann, Paris (1971)

7. Chambolle, A.: An algorithm for total variation minimization and applications. J. Math. Imaging Vis. 20, 89-97 (2004)

8. Diestel, J., Uhl, J.J.: Vector Measures. American Mathematical Society, Providence (1977)

9. Folland, G.B.: A Course in Abstract Harmonic Analysis. CRC Press, Boca Raton (1995)

10. Grupo de Inteligencia Computacional de la Universidad del País Vasco, Hyperspectral Remote Sensing Scenes. http://www.ehu.eus/ccwintco/index.php

11. Martín-Herrero, J.: Anisotropic diffusion in the hypercube. IEEE Trans. Geosci. Remote Sens. 45, 1386-1398 (2007)

12. Kunze, H., La Torre, D., Mendivil, F., Vrscay, E.R.: Fractal-Based Methods in Analysis. Springer Science \& Business Media, Berlin (2011)

13. La Torre, D., Vrscay, E.R., Ebrahimi, M., Barnsley, M.F.: Measure-valued images associated fractal transforms, and the affine self-similarity of images. SIAM J. Imaging Sci. 2, 470-507 (2009)

14. Michailovich, O., La Torre, D., Vrscay, E.R.: Function-valued mappings, total variation and compressed sensing for diffusion MRI. In: Campilho, A., Kamel, M. (eds.) ICIAR 2012, Part II. LNCS, vol. 7325, pp. 286-295. Springer, Heidelberg (2012)

15. Milman, M.: Complex interpolation and geometry of Banach spaces. Annali di Matematica Pura ed Applicata 136, 317-328 (1984)

16. Otero, D.: Function-valued Mappings and SSIM-based Optimization in Imaging, Ph.D. thesis, University of Waterloo, Waterloo, ON, Canada (2015)

17. Peetre, J.: Sur la transformation de Fourier des fonctions à valeurs vectorielles. Rendicoti del Seminario Matematico della Università di Padova 42, 15-26 (1969)

18. Thompson, H.: The Bochner Integral and an Application to Singular Integrals, M.Sc. thesis, Dalhousie University, Halifax, NS, Canada (2014)

19. Wang, Z., Bovik, A.C., Sheikh, H.R., Simoncelli, E.P.: Image quality assessment: From error visibility to structural similarity. IEEE Trans. Image Process. 13, 600-612 (2004)

20. Zeidler, E.: Nonlinear Functional Analysis and its Applications. Springer, New York (1990) 